\title{
Eficacia in vitro de la moxifloxacina frente a Candida albicans en enfermedad periodontal ${ }^{1}$
}

\section{In vitro antifungal activity of moxifloxacin against Candida isolates from periodontal disease}

\author{
ARDILA MEDINA CM* \\ ALZATE VEGA $\mathrm{J} * *$ \\ GUZMÁN ZULUAGA IC***
}

\begin{abstract}
Ardila Medina CM, Alzate Vega J, Guzmán Zuluaga IC. Eficacia in vitro de la moxifloxacina frente a Candida albicans en enfermedad periodontal. Av Periodon Implantol. 2014; 26, 1: 45-50.
\end{abstract}

\section{RESUMEN}

Introducción: La periodontitis crónica está asociada a una compleja y diversa flora microbiana que incluye posiblemente especies de Candida.

Objetivo: Evaluar la presencia de Candida y su susceptibilidad in vitro a la moxifloxacina en pacientes con periodontitis crónica.

Materiales y métodos: Se usó una técnica de diluciones seriales para evaluar la susceptibilidad de la Candida a la moxifloxacina. Se utilizó el porcentaje de individuos con al menos una bolsa infectada para describir la presencia de individuos positivos a especies de Candida.

Resultados y conclusiones: se detectaron especies de Candida en $13,2 \%$ de 76 pacientes con periodontitis crónica. La moxifloxacina inhibió significativamente el crecimiento de Candida (90\%). La moxifloxacina puede ser una buena alternativa para el tratamiento de Candida debido a que en situaciones específicas la sobreinfección por este microorganismo puede responder inadecuadamente a tratamientos periodontales convencionales.

PALABRAS CLAVE: Candida, periodontitis crónica, moxifloxacina.

\section{SUIMIMARY}

Introduction: Chronic periodontitis is associated with a widely diverse and complex subgingival microbiota including possibly Candida species.

Objective: prevalence and in vitro antifungal sensitivity of isolates of Candida species were examined in chronic periodontitis patients.

Materials and Methods: Antifungal susceptibility testing of the isolates for moxifloxacin was performed by serial dilutions technique. The presence of Candida species-positive individuals were described as the percentage of individuals with at least one infected pocket.

Results and conclusions: Candida species were detected in $13.2 \%$ of 76 chronic periodontitis patients. Moxifloxacin significantly inhibited the growth of Candida (90\%). Moxifloxacin may be a good alternative for the treatment of yeasts in periodontitis for the reason that under specific situations the superinfection by Candida can be refractory to conventional periodontal treatments.

KEYWORDS: Candida, periodontitis, moxifloxacin.

Fecha de recepción: 25 de abril de 2010.

Aceptado para publicación: 10 de junio de 2010.

Investigación financiada por la Facultad Nacional de Salud Pública y el Grupo de Epidemiología de la Universidad de Antioquía.

* Profesor Asociado Facultad de Odontología. Universidad de Antioquía. Grupo de Epidemiología. Universidad de Antioquía.

** Odontóloga. Universidad de Antioquía.

*** Periodoncista. Universidad de Chile. Profesora Asistente Facultad de Odontología Universidad de Antioquía. 


\section{INTRODUCCIÓN}

La periodontitis crónica está asociada a una diversa y compleja flora subgingival que incluye bacterias gram positivas y gram negativas, microorganismos anaerobios y facultativos, y posiblemente especies de Candida. Las especies de Candida son patógenos oportunistas que causan enfermedades en huéspedes comprometidos con procesos patológicos locales o sistémicos (1). La candidiasis es la infección micótica más común en el ser humano. En la cavidad oral, las levaduras colonizan frecuentemente la lengua, el paladar y la mucosa bucal (2) y puede observarse en placa subgingival de adultos con periodontitis (3-7). La Candida albicans es la especie de levaduras observada con mayor frecuencia (7,1-26,9\%) en periodontitis crónica (3-7). Además, la proporción de levaduras en las bolsas periodontales es similar a la de algunos periodontopatógenos, sugiriendo un posible papel de las especies de Candida en la patogénesis de la enfermedad periodontal (3-9). Las especies de Candida pueden conducir a infecciones periodontales graves en pacientes inmuno comprometidos o en individuos bajo terapia antimicrobiana por largos periodos de tiempo $(8,9)$. Bajo situaciones específicas, como en el caso de pacientes inmuno suprimidos, la sobreinfección por Candida puede ser refractaria a tratamientos periodontales convencionales $(7,9,10)$. Se sugirió también que Candida albicans puede contribuir al desarrollo de enfermedades periodontales necrotizantes en pacientes infectados con el virus de la inmuno deficiencia humana (10). Como fue anotado por Slots y cols. $(3,9)$, las especies de Candida pueden contribuir al progreso de la periodontitis y ocasionalmente pueden causar complicaciones sistémicas.

La bolsa periodontal y el fluido crevicular son ambiente favorables para la germinación y el crecimiento de hifas de Candida (7). Este tipo de hifas tienen la habilidad de penetrar los tejidos del huésped y pueden adherirse a las superficies del huésped en una mayor extensión que las levaduras (7). La Candida se ha encontrado en las capas externas de la placa bacteriana y parece que actúa como barrera entre la inmunidad del huésped y las capas internas de la biopelícula (7). De esta manera, Candida albicans puede cumplir un papel en la evasión inmune de la placa en infecciones periodontales y en la inflamación destructiva de los tejidos subyacentes (7). La pobre respuesta observada a la terapia mecánica convencional en pacientes con periodontitis crónica que presentan Candida en placa subgingival, puede deberse a sus propiedades invasivas y a su distribución en los teji- dos intraorales (11). Por estas razones, se recomienda la administración sistémica adjunta de antimicrobianos con el fin de suprimir la Candida presente en placa subgingival (9).

El desafío clínico en el tratamiento de las infecciones con levaduras se debe en parte a la acción fungistática de la mayoría de los medicamentos empleados, los cuales requieren un curso prolongado de la terapia, ocasionando efectos colaterales marcados (por ejemplo, anfotericina B) (12). Además, pruebas de susceptibilidad antifúngica, revelan alta resistencia de las especies de Candida a los azoles (13). Nuevos medicamentos antifúngicos y/o el aumento de la terapia actual pueden mejorar los resultados terapéuticos.

La moxifloxacina es una nueva 8-metoxi-quinolona oral con un amplio espectro de actividad. Es activa contra bacterias gram-positivas y gram-negativas multirresistentes, bacterias aerobias y anaerobias, y microorganismos atípicos $(14,15)$. La actividad bactericida de las fluoroquinolonas se basa principalmente en sus efectos inhibitorios sobre la $A D N$ girasa bacteriana (topoisomerasa II) (15). Las fluoroquinolonas pueden tener un efecto similar en las topoisomerasas de las levaduras (16-19). Shen y Fostel (16) fueron los primeros en sugerir la topoisomerasa II como un blanco potencial para nuevos agentes antifúngicos. Otros informes indican potenciación por parte de las fluoroquinolonas en la actividad antifúngica de la anfotericina $\mathrm{B}$ y el fluconazol, tanto in vivo como in vitro $(18,19)$.

De esta manera, el objetivo de este estudio fue evaluar la actividad antifúngica de la moxifloxacina frente a especies de Candida presentes en placa subgingival de pacientes con periodontitis crónica.

\section{MATERIALES Y MÉTODOS}

\section{Sujetos}

En este estudio se invitaron a participar 76 sujetos sistémicamente sanos ( 45 mujeres y 31 hombres) que asistieron a las clínicas odontológicas de la Facultad de Odontología de la Universidad de Antioquía entre octubre de 2008 y marzo de 2009. Cada participante firmó un consentimiento informado. El Comité de Ética de la Sede de Investigación Universitaria de la Universidad de Antioquía aprobó el diseño del estudio teniendo en cuenta la Declaración de Helsinki sobre experimentación que involucra seres humanos. Para participar en esta investigación, se consideraron 
como candidatos, pacientes con diagnóstico de periodontitis crónica. Los criterios de exclusión fueron embarazo, lactancia, presencia de diabetes o cualquier enfermedad sistémica que alterara el curso de la enfermedad periodontal, terapia periodontal en el último año, y utilización de antimicrobianos o antiinflamatorios no esteroides en los seis meses previos al examen clínico y a la toma de muestras microbiológicas.

\section{Evaluación clínica}

A cada paciente se le realizó una historia clínica, además de un examen clínico y radiográfico completo. Uno de los autores (CA) realizó todos los exámenes clínicos. La profundidad de sondaje (PS), el nivel de inserción clínica (NIC), y la presencia de sangrado, supuración y placa se midieron en seis sitios (mesiobucal, bucal, distobucal, distolingual, lingual y mesiolingual) usando una sonda periodontal calibrada (UNC-15, Hu-Friedy, Chicago, IL). El diagnóstico de periodontitis crónica se realizó según los criterios recomendados por la Academia Americana de Periodoncia (AAP) (20).

\section{Muestreo microbiológico}

Se tomaron muestras microbiológicas de los pacientes en sitios con una profundidad de sondaje $\geq 5 \mathrm{~mm}$. Para el muestreo se seleccionaron las seis bolsas periodontales más profundas. Después de aislar la zona con algodón y remover la placa supragingival con cureta, se insertaron conos de papel estéril en cada bolsa periodontal durante 20 segundos. Las muestras de cada paciente se depositaron en $2 \mathrm{~mL}$ de medio de transporte (Viability Medium Göteborg Anaerobically III: VMGA III) (21) y se llevaron al laboratorio de microbiología de la Facultad de Odontología de la Universidad de Antioquía para procesarlas dentro de las dos horas siguientes.

Cultivo de levaduras: Las muestras fueron inoculadas en agar dextrosa de Saboraud (ADS) y fueron inoculadas también en Agar HiCrome diferencial (agar CHROM) (Hi Media Pvt. Ltd., Mumbai) para mejorar la identificación de las especies, teniendo presente la morfología de la colonia coloreada. Las placas de ADS y de agar CHROM se incubaron a $37^{\circ} \mathrm{C}$ durante 48 horas. Los aislamientos se caracterizaron por la realización de las siguientes pruebas: producción tubo germinal, formación de clamidosporas en agar harina de maíz, perfil de la asimilación de azúcar y el crecimiento diferencial en $\mathrm{ADS}$ a $37^{\circ} \mathrm{C}$ y $42^{\circ} \mathrm{C}(22,23)$. Para cada aislamiento se preparó un inoculo en una solución salina estéril y se ajustó a un patrón de turbidez $0,5 \mathrm{McFarland}\left(10^{8} \mathrm{ufc} / \mathrm{ml}\right)$ utilizando un espectrofotómetro.

\section{Pruebas de sensibilidad antifúngica}

Para la evaluación in vitro de la actividad antifúngica de la moxifloxacina se utilizaron los aislamientos de Candida recuperados de placa subgingival. Las pruebas de susceptibilidad antifúngica de los aislamientos se realizaron mediante la técnica de diluciones seriadas de acuerdo con el Comité Nacional para Normas del Laboratorio Clínico (NCCLS) (24). Se preparó moxifloxacina sin diluir y también se prepararon diluciones en serie de la preparación comercial. Se obtuvieron tres diluciones de moxifloxacina al 0,5\%, 0,25\% y $0,125 \%$. Se inocularon tres tubos, cada uno con $1 \mathrm{ml}$ de 3 diluciones de moxifloxacina, con $50 \mu \mathrm{l}$ de 0,5 McFarland. Los tubos se incubaron a $35^{\circ} \mathrm{C}$ durante 24 horas. Las alícuotas se sembraron en agar Sabouraud y se incubaron a $35^{\circ} \mathrm{C}$ durante 24 horas. Las colonias se contaron y registraron a las 24 horas.

\section{Análisis estadístico}

Para describir las variables relacionadas con los sujetos y los dientes se realizó un análisis exploratorio sobre la distribución de los índices PS y NIC utilizando medidas de tendencia central y de dispersión. Se obtuvieron frecuencias y proporciones de las especies de Candida. Para las variables placa bacteriana, sangrado al sondaje, y supuración se calcularon frecuencias y proporciones. Se utilizó la prueba de chicuadrado para evaluar la diferencia entre presencia de sangrado frente a la presencia o ausencia de Candida.También se usó la prueba de Mann-Whitney para determinar las diferencias en la PS y el NIC en presencia o ausencia de Candida. Se usó un programa estadístico para todos los análisis (SPSS, versión 15, Chicago, IL).

\section{RESULTADOS}

Se estudiaron 31 hombres (41\%) y 45 mujeres (59\%) con periodontitis crónica (edad: $46 \pm 8,1$ años), de los cuales fumaban el $22 \%$. Las tablas 1 y 2 presentan las características clínicas y demográficas de los pacien- 


\section{TABLA 1.- CARACTERÍSTICASS DEMOGRÁFICÁS DE PACIENTES CON PRESENCIA DE CANDIDA}

\begin{tabular}{|l|c|}
\hline Parámetro & $\begin{array}{c}\text { Presencia } \\
\text { de Candida }\end{array}$ \\
\hline $\mathrm{n}$ & 10 \\
$\%$ & 13,2 \\
Edad (años $\pm \mathrm{DE})$ & $39,6 \pm 5,9$ \\
Género & $70 \%$ \\
- \% mujeres & $30 \%$ \\
- \%ábito de fumar & \\
• \% no-fumadores & $70 \%$ \\
- \% fumadores & $30 \%$ \\
\hline DE: desviación estándar. & \\
\hline
\end{tabular}

\begin{tabular}{|c|c|}
\hline $\begin{array}{r}\text { TABLA 2.- CARACTERÍS } \\
\text { CLÍNICAS DE PACIENTE } \\
\text { PRESENCIA DE CANDIDA } \\
\text { SUBGINGIVAL }\end{array}$ & $\begin{array}{l}\text { IICAS } \\
\text { CON } \\
\text { N PLACA }\end{array}$ \\
\hline Parámetro & $\begin{array}{c}\text { Presencia } \\
\text { de Candida }\end{array}$ \\
\hline $\begin{array}{l}\text { Profundidad de sondaje }(\mathrm{mm} \pm \mathrm{DE}) \\
\text { Nivel de inserción clínica }(\mathrm{mm} \pm \mathrm{DE}) \\
\% \text { sangrado al sondaje }(\% \pm \mathrm{DE}) \\
\% \text { placa bacteriana }(\% \pm \mathrm{DE}) \\
\% \text { supuración }(\% \pm \mathrm{DE})\end{array}$ & $\begin{array}{c}3 \pm 1,2 \\
3,8 \pm 1,7 \\
69 \pm 21 \\
46 \pm 28 \\
14 \pm 3,1\end{array}$ \\
\hline
\end{tabular}

tes con presencia de Candida. Se observaron especies de Candida en 10 (13,2\%, Tabla 1$)$ de 76 pacientes con periodontitis crónica. Las mujeres $(7 / 45 ; 15,8 \%)$ presentaron más Candida que los hombres $(3 / 31 ; 9,8 \%$, $\mathrm{p}<0,001)$. Se observó Candida albicans en 8 pacientes y en dos sujetos se encontraron dos especies sin especificar. Las pruebas de microdilución de la moxifloxacina mostraron una inhibición significativa del crecimiento de Candida (90\%) en todas las concentraciones no diluidas. No hubo crecimiento de los organismos en el control estéril.

\section{DISCUSIÓN}

En el presente estudio se identificaron especies de Candida en placa subgingival en el $13,2 \%$ de 76 pacientes con periodontitis crónica. Slots y colaboradores (9) observaron frecuencias similares (13-14\%) a las encontradas en esta investigación. En el estudio de Slots y colaboradores (9) todos los pacientes fueron sometidos a terapia mecánica convencional y la mayoría de ellos recibieron uno o más tratamientos con antibióticos a corto plazo. Es importante tener presente que en ese estudio todos los sitios de muestreo fueron definidos como no respondedores o refractarios a la terapia periodontal. Otro estudio realizado por Slots y colaboradores (3) también registró $16,8 \%$ de bolsas periodontales infectadas por levaduras en pacientes con pobre respuesta a la terapia periodontal. Al igual que en el presente estudio la levadura más frecuentemente fue C. albicans. El alto potencial patogénico de estos microorganismos (3) puede representar una causa de fracaso en la terapia periodontal $(7,9,10)$. Se desconoce el papel de las especies de Candida en la patogénesis de la periodontitis, pero algunos investigadores sugieren que estos patógenos pueden tener gran impacto en el progreso y tratamiento de la enfermedad periodontal $(3,7,9,10)$. Como ha señalado Slots y cols. (25), las levaduras persisten después de terapia mecánica y quirúrgica. Se requieren estudios adicionales para evaluar los factores de riesgo, los mecanismos de virulencia, y el impacto del tratamiento en pacientes que presenten Candida en placa subgingival.

La susceptibilidad antimicrobiana obtenida en el presente estudio sugiere que la moxifloxacina tiene el potencial para erradicar especies de Candida. La moxifloxacina es una nueva fluoroquinolona de amplio espectro incluyendo anaerobios y microorganismos gram-positivos, especialmente aquellos multirresistentes (26). Presenta además excelente biodisponibilidad, a largo y mediano plazo, buena penetración tisular (27) y tolerancia (28).

En el presente estudio la prueba de microdilución reveló que la actividad antifúngica fue más evidente con concentraciones de moxifloxacina sin diluir: $0,5 \%(0,5$ $\mathrm{mg} / \mathrm{ml}$ ), inhibiendo significativamente el crecimiento de casi todas las especies de Candida. Sin embargo, las actividades de los fármacos in vivo pueden ser diversas, lo que impide realizar cualquier conclusión sobre la eficacia clínica.

Son pocos los estudios previos que sugieren una actividad antifúngica de las fluoroquinolonas (16-19, 29). 
Sin embargo, otros antibacterianos como la minociclina también presentaron actividad antifúngica en varios estudios (30-32).

Recientemente, la FDA aceptó la incorporación de 22 agentes patógenos a la eficacia in vitro de la moxifloxacina, incluyendo muchas micobacterias atípicas (29). Las fluoroquinolonas tienen actividad bactericida que se determina principalmente por su actividad contra el ADN girasa (topoisomerasa II) y la topoisomerasa IV, dos enzimas con funciones esenciales en la síntesis de $A D N(16-17)$. Los principales hongos patógenos tienen altos niveles de topoisomerasa II, lo cual permite explicar la eficacia in vitro de la moxifloxacina frente a especies de Candida (18).

\section{CONCLUSIONES}

Su buena actividad contra las especies de Candida sugiere el uso potencial de la moxifloxacina como antibiótico adjunto al tratamiento de infecciones mixtas periodontales. La realización de estudios que incluyan una mayor cantidad de aislamientos, podría permitir el desarrollo de fluoroquinolonas antifúngicas dirigidas particularmente a las topoisomerasas de las levaduras. El buen resultado obtenido por la moxifloxacina puede ser un indicio para realizar pruebas de este antibiótico en ensayos clínicos controlados.

\section{BIBLIOGRAFÍA}

1. McCullough MJ, Ross BC, Reade PC. Candida albicans: a review of its history, taxonomy, epidemiology, virulence attributes, and methods of strain differentiation. Int J Oral Maxillofac Surg 1996;25:136-44.

2. Hannula J, Dogan B, Slots J, Okte E, Asikainen S. Subgingival strains of Candida albicans in relation to geographical origin and occurrence of periodontal pathogenic bacteria. Oral Microbiol Immunol 2001;16: 113-18.

3. Slots J, Rams TE, Listgarten MA. Yeasts, enteric rods and pseudomonads in the subgingival flora of severe adult periodontitis. Oral Microbiol Immunol 1988;3:47-52.

4. Reynaud AH, Nygaard-Østby B, Bøygard G-K, Eribe ER, Olsen I, Gjermo P. Yeasts in periodontal pockets. J Clin Periodontol 2001;28: 860-4.

5. Urzúa B, Hermosilla G, Gamonal J, Morales-Bozo I, Canals $\mathrm{M}$, Barahona S, and others. Yeast diversity in the oral microbiota of subjects with periodontitis: Candida albicans and Candida dubliniensis colonize the periodontal pockets. Med Mycol 2008; 46:783-93.

6. Papapanou PN. Population studies of microbial ecology in periodontal health and disease. Ann Periodontol 2002;7:54-61.

7. Jarvensivu A, Hietanen J, Rautemaa R, Sorsa T, Richardson M. Candida yeasts in chronic periodontitis tissues and subgingival microbial biofilms in vivo. Oral Dis 2004;10:106-12.

8. Helovuo H, Hakkarainen K, Paunio K. Changes in the prevalence of subgingival enteric rods, staphylococci and yeasts after treatment with penicillin and erythromycin. Oral Microbiol Immunol 1993;8:75-9.

9. Slots J, Feik D, Rams TE. Age and sex relationships of superinfecting microorganisms in periodontitis patients. Oral Microbiol Immunol 1990;5:305-8.

10. Brawner DL, Cuttler JE. Oral Candida albicans isolates from nonhospitalized normal carriers, immunocompetent hospitalized patients, and immunocompromised patients with or without acquired immunodeficiency syndrome. J Clin Microbiol 1989;27:1335-41.

11. Walker C, Karpinia K. Rationale for use of antibiotics in periodontics. J Periodontol 2002; 73:1 188-96.

12. Marangon FB, Miller D, Giaconi JA, Alfonso EC. In vitro investigation of voriconazole susceptibility for keratitis and endophthalmitis fungal pathogens. Am J Ophthalmol 2004;137:820-5.

13. Chunchanur SK, Nadgir SD, Halesh LH, Patil BS, Kausar Y, Chandrasekhar MR. Detection and antifungal susceptibility testing of oral Candida dubliniensis from human immunodeficiency virus-infected patients. Indian J Pathol Microbiol 2009;52:501-4.

14. Guentsch A, Jentsch H, Pfister W, Hoffmann T, Eick S. Moxifloxacin as an adjunctive antibiotic in the treatment of severe chronic periodontitis. J Periodontol 2008; 79:1894-3.

15. Eick S, Pfister W. Efficacy of antibiotics against periodontopathogenic bacteria within epithelial cells: an in vitro study. J Periodontol 2004; 75: 1327-34.

16. Shen L, Mitscher LA, Sharma PN, O'Donnell TJ, Chu DW, Cooper CS, and others. Mechanism of inhibition of DNA 
gyrase by quinolone antibacterials. A cooperative drugDNA binding model. Biochemistry 1989;28:2886-94 .

17. Shen LL, Fostel JM. DNA topoisomerase inhibitors as antifungal agents. Adv Pharmacol1994;29B: 227-44.

18. Stergiopoulou T, Meletiadis J, Sein T, Papaioannidou P, Tsiouris I, Roilides E, et al. Comparative pharmacodynamic interaction analysis between ciprofloxacin, moxifloxacin and levofloxacin and antifungal agents against Candida albicans and Aspergillus fumigatus. J Antimicrob Chemother 2009;63:343-8.

19. Sugar AM, Liu XP, Chen RJ. Effectiveness of quinolone antibiotics in modulating the effects of antifungal drugs. Antimicrob Agents Chemother 1997;41:2518-21.

20. Armitage GC. Development of a classification system for periodontal diseases and conditions. Ann Periodontol 1999; 4:1-6.

21. Möller AJ. Microbiological examination of root canals and periapical tissues of human teeth. Methodological studies. Odontol Tidskr 1966; 74:Suppl:1-380.

22. Khan ZU, Ahmad S, Mokaddas E, Al-Sweih N, Chandy R. Sunflower seed husk agar: a new medium for the differentiation of Candida dubliniensis from Candida albicans. Indian J Med Microbiol 2005;23:182-5.

23. Marot-Leblond A, Grimaud L, David S, Sullivan DJ, Coleman DC, Ponton J, et al. Evaluation of a rapid immunochromatographic assay for identification of Candida albicans and Candida dubliniensis. J Clin Microbiol 2004;42:4956-60.

24. National Committee for Clinical Laboratory Standards. 1997. Reference method for dilution antifungal susceptibility testing of yeasts; Approved standard NCCLS document M27-A. National Committee for Clinical Laboratory Standards, Wayne, Pa.

25. Slots J, Rams TE, Feik D, Taveras HD, Gillespie GM. Subgingival microflora of advanced periodontitis in the Dominican Republic. J Periodontol 1991;62:5437 .

26. Cachovan G, Nergiz I, Thuss U, Siefert HM, Sobottka I, Oral O, and others. Penetration of moxifloxacin into rat mandibular bone and soft tissue. Acta Odontol Scand 2009; 67:182-6

27. Krasemann C, Meyer J, Tillotson G. Evaluation of the clinical microbiology profile of moxifloxacin. Clin Infect Dis 2001; 32 Suppl 1:S51-63.

28. Mosca A, Miragliotta L, Iodice MA, Abbinante A, Miragliotta G. Antimicrobial profiles of Prevotella spp. and Fusobacterium nucleatum isolated from periodontal infections in a selected area of southern Italy. Int J Antimicrob Agents 2007; 30: 521-4.

29. Ozdek SC, Miller D, Flynn PM, Flynn HW. In vitro antifungal activity of the fourth generation fluoroquinolones against Candida isolates from human ocular infections. Ocul Immunol Inflamm 2006;14:347-51.

30. Lew MA, Beckett KM, Levin MJ. Antifungal activity of four tetracycline analogues against Candida albicans in vitro: Potentiation by amphotericin B. J Infect Dis 1977;136:26370.

31. Schierholz JM, Pulverer G, Bach A, Wachol-Drebeck Z. In vitro activity of rifampin-minocyclin coating to Candida albicans. Crit Care Med 1999;27:1691-3.

32. Wilson M, O'Connor B, Newman HN. Isolation and identification of bacteria from subgingival plaque, with low susceptibility to minocycline.J Periodontol 1991;28: 71-8.

\section{CORRESPONDENCIA}

Carlos M. Ardila Medina

Calle $64 \mathrm{~N}^{\circ}$ 52-59

Medellín, Colombia

e-mail: martinardila@gmail.com. Fax: 057-4-2195332 